\title{
Management implication on Indian Ocean albacore from simple yield analysis incorporating parameter uncertainty
}

\author{
Shui-Kai Chang ${ }^{\mathrm{a}}$, Chien-Chung Hsu ${ }^{\mathrm{b}, *}$, Hsi-Chiang Liu ${ }^{\mathrm{b}}$ \\ ${ }^{a}$ Overseas Fisheries Development Council, 19, Lane 113, Roosevelt Road, Sec. 4, Taipei 106, Taiwan \\ ${ }^{\mathrm{b}}$ Institute of Oceanography, National Taiwan University, P.O. Box 23-13, Taipei 100, Taiwan
}

Received 28 July 1999; received in revised form 23 May 2000; accepted 12 August 2000

\begin{abstract}
Many studies have been conducted to estimate the parameters of Indian Ocean albacore (Thunnus alalunga) fishery. Various estimates on the same parameter from these studies have resulted in uncertainty and confusion when management strategies are made. This study incorporates these estimates in the simple yield-per-recruit model through Monte Carlo simulations to derive management implication under parameter uncertainty.

The results provided an optimal management suggestion under the following conditions. First, the probability of obtaining feasible simulation results was greater than 95\%; second, the probability of increase to yield-per-recruit after regulatory change was also greater than $95 \%$. Under these conditions, the results suggested if the goal of management was to increase the relative yield-per-recruit of the Indian Ocean albacore stock by $30 \%$ and more, the relative selection size should be changed by +0.15 to +0.25 and the exploitation rate by -0.5 to +0.15 . (C) 2001 Elsevier Science B.V. All rights reserved.
\end{abstract}

Keywords: Indian albacore management; Parameter uncertainty; Simple yield-per-recruit model

\section{Introduction}

Indian Ocean albacore (Thunnus alalunga) has been taken by longline fishery since the 1950s and by gillnet fishery since 1985, but gillnetting was banned in end of 1992. Japan, Korea and Taiwan were the leading longline nations fishing albacore in the Indian Ocean before 1976, and now, Taiwan is the only one using longline to target this species (Chang, 1993). Ever since this stock was exploited, many studies (Lee and Liu, 1992; Chang, 1993; Chang et al., 1993) have been conducted to understand its biological characteristics and status, and provide information on how best to

\footnotetext{
* Corresponding author. Tel.: +886-2-362-2987; fax: +886-2-366-1198.

E-mail address: hsucc@ccms.ntu.edu.tw (C.-C. Hsu).
}

manage this fishery. However, depending on the nature of the used data and the computation approaches, several different sets of parameter estimates have been derived (Table 1) and it is not easy to select the most appropriate one for management purposes. For example, using different age characters (scale or otolith), different back-calculation formulae (direct proportional or second-order parabolic), different fitting methods (Walford's graphic or nonlinear fitting), and data of different time-spans have provided quite different estimates on growth parameters $\left(K, L_{\infty}\right)$ (Chang and Hsu, 1992). Thus, there were altogether six sets of growth parameter estimates derived or recalculated for this stock in the past (Table 1).

Besides the variation in estimates of the same parameter, each estimate itself has involved various types of variance; it may also inherit some bias 
Table 1

The growth parameters, natural and fishing mortalities of Indian Ocean albacore estimated in previous studies

\begin{tabular}{|c|c|c|c|c|c|c|}
\hline \multirow[t]{2}{*}{ Sources, age characters used } & \multirow[t]{2}{*}{ Data note $^{a}$} & \multirow[t]{2}{*}{ Fitting method } & \multirow[t]{2}{*}{$K$} & \multirow[t]{2}{*}{$L_{\infty}$} & \multicolumn{2}{|c|}{ Length $^{\mathrm{b}}$} \\
\hline & & & & & Age-1 & Age-2 \\
\hline \multicolumn{7}{|l|}{ Growth parameters } \\
\hline Huang et al. $(1990)^{\mathrm{c}}$, scale & LL and GG, 1987-1990 & Walford & 0.162 & 128.1 & 33.9 & 48.0 \\
\hline Huang et al. (1990) ${ }^{\mathrm{d}}$, scale & LL and GG, $1987-1990$ & Nonlinear & 0.149 & 132.2 & 36.6 & 49.9 \\
\hline Lee and Liu (1992), vertebrae & LL and GG, 1986-1989 & Walford & 0.109 & 163.7 & 46.5 & 58.6 \\
\hline Lee and Liu (1992) $)^{\mathrm{d}}$, vertebrae & LL and GG, 1986-1989 & Nonlinear & 0.097 & 167.1 & 44.2 & 55.6 \\
\hline Chang et al. (1993), length frequency & LL, 1983-1986 & MULTIFAN & 0.118 & 171.4 & 43.8 & 58.0 \\
\hline Chang et al. (1993), length frequency & LL, 1988-1990, when GG thriving & MULTIFAN & 0.133 & 147.2 & 41.6 & 54.8 \\
\hline Source & Method & $M$ & & & & \\
\hline \multicolumn{7}{|l|}{ Natural mortality } \\
\hline Lee et al. $(1991)^{\mathrm{e}}$ & Pauly's empirical equation & 0.206 & & & & \\
\hline Liu and Lee (1992) & Linear fitting on $Z=q f+M$ & 0.221 & & & & \\
\hline Chang et al. (1993) & MULTIFAN & $0.22-0.25$ & & & & \\
\hline Source & 1988 & 1989 & 1990 & & & \\
\hline \multicolumn{7}{|c|}{ Fishing mortality (estimates of each study's last few years) } \\
\hline Liu and Lee (1992) & 0.33 & - & - & & & \\
\hline Chang (1993) & 0.43 & 0.33 & 0.28 & & & \\
\hline
\end{tabular}

${ }^{a}$ LL: longline fishery; GG: gillnet fishery.

${ }^{\mathrm{b}}$ Estimated mean length $(\mathrm{cm})$ at age from the specific study.

${ }^{c}$ Huang, C.-S., Wu, C.-L., Kuo, C.-L., Su, W.-C., 1990. Age and growth of the Indian Ocean albacore, Thunnus alalunga, by scales. IPTP Working Paper, IPTP/TWS/90/53, pp. 111-122.

${ }^{\mathrm{d}}$ Re-calculated using nonlinear fitting approach by Chang et al. (1993).

${ }^{\mathrm{e}}$ Lee, Y.-C., Hsu, C.-C., Chang, S.-K., Liu, H.-C., 1991. Yield-per-recruit analysis of the Indian Ocean albacore stock. IPTP Coll. Vol. Work. Doc. 4, pp. 136-148.

(Hilborn and Walters, 1992). Here we simply refer to different estimates of a parameter and the variance of the estimates themselves as the "parameter uncertainty". This uncertainty is inevitable in the parameter estimation of dynamic fishery systems, and needs to be dealt with while making fishery management proposals. The importance on this aspect has often been emphasized (Hilborn, 1979; Ludwig, 1981; Majkowski and Hampton, 1983; Restrepo and Fox, 1988; Hilborn and Walters, 1992).

To provide scientific advice on the efficient use of the albacore stock, the yield-per-recruit model (Beverton and Holt, 1957) is widely used for management purpose (Liu and Lee, 1992). Therefore, the objective of this study is to assume a probability distribution on the different parameter estimates reported previously (Table 1), and to use Monte Carlo technique to randomly select values from the distribution (Restrepo and Fox, 1988). Then, we select those values as the "input parameters with uncertainty" to compute the simple yield-per-recruit. Finally, the simulation results derived from yield-per-recruit model can be used to draw the management implication for the stock.

\section{Materials and methods}

\subsection{The simple yield-per-recruit model and the input parameters}

The yield-per-recruit model was used in this study to examine management implications for the albacore stock. The original complete form of the model (Beverton and Holt, 1957) contains at least seven parameters. Let $t_{\mathrm{r}}$ and $t_{\mathrm{c}}$ denote the age of recruitment to the fishery and age of first catch; $F$ and $M$ the fishing mortality and the natural mortality; $W_{\infty}, K$ and $t_{0}$ the parameters of von Bertalanffy growth equation. Then, provided that (1) the exploitation rate $E$ equals $E=F /(F+M) ;$ (2) the mean selection length 
(mean length at first capture) $l_{\mathrm{c}}$ equals $l_{\mathrm{c}}=L_{\infty}$ $\left(1-\mathrm{e}^{-K\left(t_{\mathrm{c}}-t_{0}\right)}\right)$; (3) the arbitrary recruitment $R_{0}$ at age $t_{0}$ equals $R_{0}=R \mathrm{e}^{M\left(t_{\mathrm{r}}-t_{0}\right)}$, the original yield equation can be simplified as

$$
\begin{aligned}
Y^{\prime} & =\frac{Y}{R_{0} W_{\infty}} \\
& =E(1-C)^{M / K} \sum_{n=0}^{3} \frac{U_{n}(1-C)^{n}}{1+n(M / K)^{-1}(1-E)},
\end{aligned}
$$

where $U_{n}$ is summation variable, taking values $1,-3$, $3,-1$ for $n=0,1,2,3$, respectively; $C$ the mean length at first catch relative to maximum length or the relative selection size $\left(=l_{\mathrm{c}} / L_{\infty}\right), Y^{\prime}$ the equilibrium relative yield-per-recruit (independent of units). There are only three parameters left, i.e., $E, C$, and $M / K$ in this simplified equation, and they are "input parameters" to the model in the present study.

\subsection{Uncertainty distribution of the input parameters}

To investigate the parameter uncertainty on the estimates of each input parameter, distributions to elucidate uncertainty are assumed and assigned to each parameter. According to the assuming distribution, the input values can be randomly selected for computation during the Monte Carlo simulation. Two of the useful probability distributions are uniform and triangular distributions (Restrepo and Fox, 1988). Uniform distribution assumes that the values within the estimate ranges of each parameter have equal possibility to be the "true" parameter values. That is, the continuous random input value $(x)$ will be picked up with equal possibility from the given ranges $[\alpha, \beta]$ for the simulation runs, where $\alpha$ and $\beta$ are the lower and upper estimates of parameter. The probability density function (p.d.f.) of $x$ that have a uniform distribution is

$f(x ; \alpha, \beta)= \begin{cases}\frac{1}{\beta-\alpha}, & \alpha \leq x \leq \beta, \\ 0, & \text { otherwise }\end{cases}$

On the other hand, triangular distribution assumes that some specific values in the ranges are more likely to be the "true" parameter values. If there were no clear indications which value is more likely to be the "true" value than the others, uniform distribution would be a good choice. However, if there was, based on the best "knowledge", one value with the highest possibility (the mode) to be the "true" value, then the triangular distribution would be better adopted. The p.d.f. of a continuous random value to have a triangular distribution is

$f(x ; \alpha, \beta, \gamma)= \begin{cases}\sqrt{\frac{\gamma-\alpha}{\beta-\alpha} U(0,1)}, & x \leq \frac{\gamma-\alpha}{\beta-\alpha}, \\ 1-\sqrt{\frac{\beta-\gamma}{\beta-\alpha}[1-U(0,1)]}, & \text { otherwise }\end{cases}$

where $\gamma$ is the mode of the distribution and $U(0,1)$ the uniform distribution on the interval of $[0,1]$.

For the target stock of this study, many estimations on its fishery parameters have been done in the past (Table 1). These parameter estimates ranged but with a plausible "mode" when considering the quality of the estimation itself (discussed below). In this regard, the present study was conducted intensively using the triangle distribution as the input uncertainty distribution since some more plausible input values might be obtained from the previous estimations. The ranges and mode values provided in the following section were used for the simulation runs. Uniform distribution was also applied latter for sensitivity comparison.

\subsection{Specific values and their ranges of the input parameters}

Basically, there are four fishery parameters to be calculated to obtain the input parameters, i.e., growth parameters $\left(K, L_{\infty}\right)$, natural mortality $(M)$, fishing mortality $(F)$ and mean selection length $\left(l_{\mathrm{c}}\right)$. For Indian Ocean albacore, the first three parameters have already been estimated in past studies (Lee and Liu, 1992; Liu and Lee, 1992; Chang, 1993; Chang et al., 1993) and are listed in Table 1. There are altogether six sets of growth parameter estimates, three $M_{\mathrm{s}}$ and two possible $F_{\mathrm{s}}$ for the recent years.

As mentioned previously, there were two albacore fisheries in the Indian Ocean in the past: longline fishery and gillnet fishery. The former mainly utilized fish starting from age one to two, and the latter, which is now banned, utilized fish from age one. According to Table 1 , the $l_{\mathrm{c}}$ is thus assumed to be in the range of $34-59 \mathrm{~cm}$, depending on the estimation approaches. Our experience in collecting the albacore size data (Fig. 2 in Chang et al., 1993) suggested that the most 
plausible selection length might be somewhere between 40 and $50 \mathrm{~cm}$.

From Table 1, the estimate range of each input parameter can be summarized as $C: 0.20-0.46 ; E$ : $0.41-0.88 ; M / K: 1.27-2.58$. Based on the available information, each parameter could be specified intuitively with a value as its most possible "true" value. Taking $K$ as an example, we have not much confidence on the estimation result from scale studies because of some of its deficiencies (Casselman, 1983; Carlander, 1987), nor on the result from length data when gillnet fishery was thriving (Chang et al., 1993). Therefore, from the rest estimation results, it could be considered that the "true" or most plausible value might be somewhere between 0.097 and 0.118 , and that the most possible one might be around 0.11 .

The most likely values thus specified in the present study are $C: 0.27 ; E: 0.68 ; M / K: 2.0$. The reason for specifying these ranges and the most possible values is to satisfy the requirements of the input uncertainty distribution discussed.

\subsection{Index for examining the simulation results}

Since $Y^{\prime}$ itself is a function of $M / K$ and $M / K$ is not fixed, the $Y^{\prime}$ in Eq. (1) cannot be used directly to examine the effect of uncertainty (Beverton and Holt, 1966; Restrepo and Fox, 1988). The percent change instead is used in the present study to examine the fraction gains or losses of $Y^{\prime}$ due to the changes in $E$ or $C$. The $E$ and $C$ are referred as the "control parameters" of the computation. Such percentage change is regarded as the simulation of management regulation, i.e., changing of exploitation rate to the stock and changing in relative selection size. The percent change of $Y^{\prime}$ (PCYR), is defined as

$\operatorname{PCYR}=\frac{Y_{\mathrm{a}}^{\prime}-Y_{\mathrm{b}}^{\prime}}{Y_{\mathrm{b}}^{\prime}} \times 100$,

where $Y_{\mathrm{b}}^{\prime}$ and $Y_{\mathrm{a}}^{\prime}$ are the $Y^{\prime}$ before and after the control parameters are changed (due to regulation), respectively.

\subsection{Infeasible results}

In this study, we added to or subtracted from the control parameters $(C$ and $E$ ) a quantity to simulate a regulatory change. By definition, $E$ and $C$ must be in the range of $0-1$. However, during the simulation runs, it is possible that the randomly selected values of $E$ or $C$ after adding or subtracting process may be beyond the valid bounds. Many such occurrences might be noted in the case of initial uncertainty distribution for $E$ or $C$ consists of high values and thus this distribution is shifted upwards (Restrepo and Fox, 1988). Any conclusion drawn from such computations may be meaningless. Therefore, we ignored them during the simulation and referred to such invalid values as "infeasible results". The percentage of infeasible results is defined here as results of $[$ infeasible $/($ infeasible + feasible $)] \times 100$.

\section{Results}

For the first part, we simulated the regulation of increasing exploitation rate by 0.1 while the relative selection size was kept at the current level; i.e., increasing $E$ by adding 0.1 to its input and $C$ remained unchanged. The input uncertainty distribution was assumed triangular. The result of 600 simulation runs (Fig. 1) shows that most PCYR happened in the range of -4 to $-17 \%$, with a mean of $-10.6 \%$ and standard deviation of $7.4 \%$. This indicates that if we adopt the regulation, then, with the uncertainty in the input parameters, there would be $90 \%$ probability that PCYR would decrease and about $70 \%$ of the decrease would be around $4-17 \%$.

For a real situation of management, the regulation option might be adopted to change both $E$ and $C$. Therefore, we performed 4000 simulation runs on each combination of the increment in $E(-0.3$ to $+0.3)$ and $C(+0.0$ to +0.6$)$, and obtained a contour diagram of the output PCYR from the simulation results. There are two points noted from Fig. 2, shows the isopleth of mean PCYR. First, at current uncertainty, when we increased $C$ by an increment of $0.2-0.4$ and increased $E$ by as high as 0.3 , we could still produce $40 \%$ increase of PCYR in average (hatched lines in Fig. 2). Second, the roughly horizontal oval shape of the contour lines implies that the change in $C$ might have higher efficiency in the increase of PCYR than the change in $E$.

The percentage of "positive" PCYR in the simulation results is plotted in Fig. 3. The position of higher percentage represents those combinations of changes 


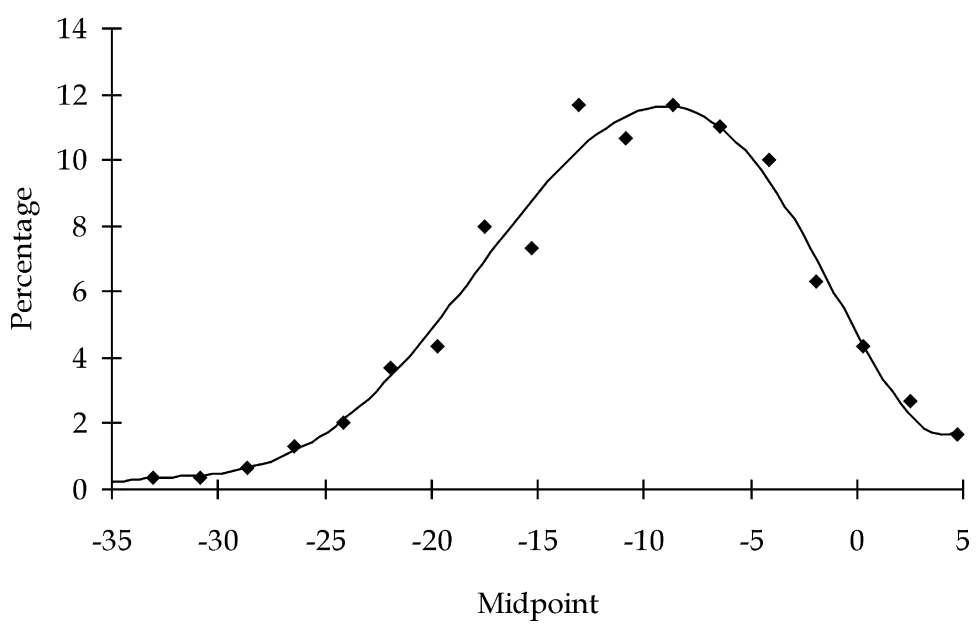

Fig. 1. Distribution of PCYR of 600 simulation runs, under the condition of control parameter $E$ increased by 0.1 and $C$ remained unchanged. Dots in the map are the percentage of PCYR, and the line is their polynomial-fitted curve.

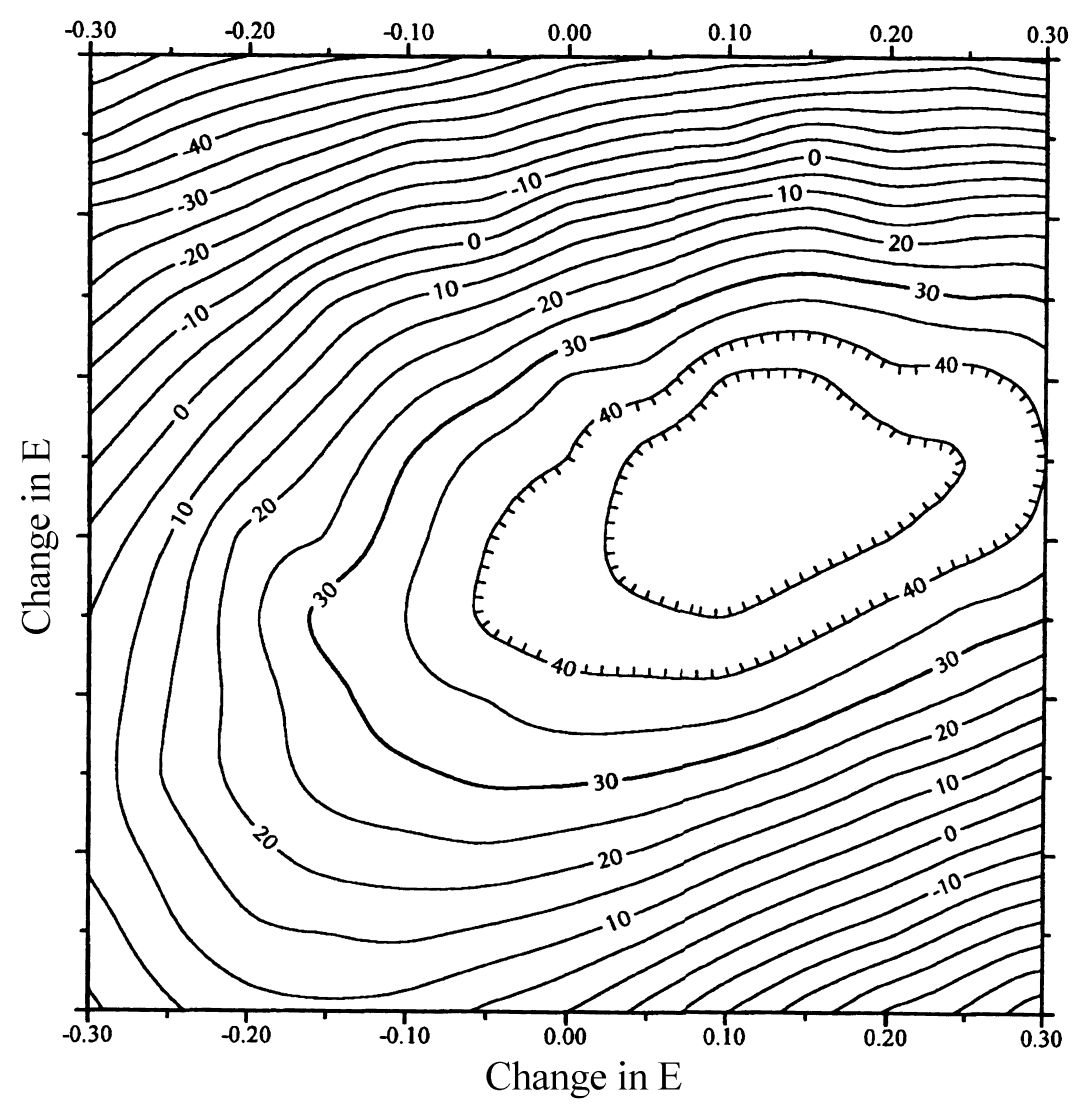

Fig. 2. Isopleth of mean PCYR outputs from 4000 simulation runs for various combinations of changes in $E$ and $C$. Hatched lines indicate the contour lines with mean PCYR greater than $40 \%$, and darker line indicates the reference line to be used in Fig. 4. 


\section{Percentage of Positive PCYR (Triangular input dist.)}

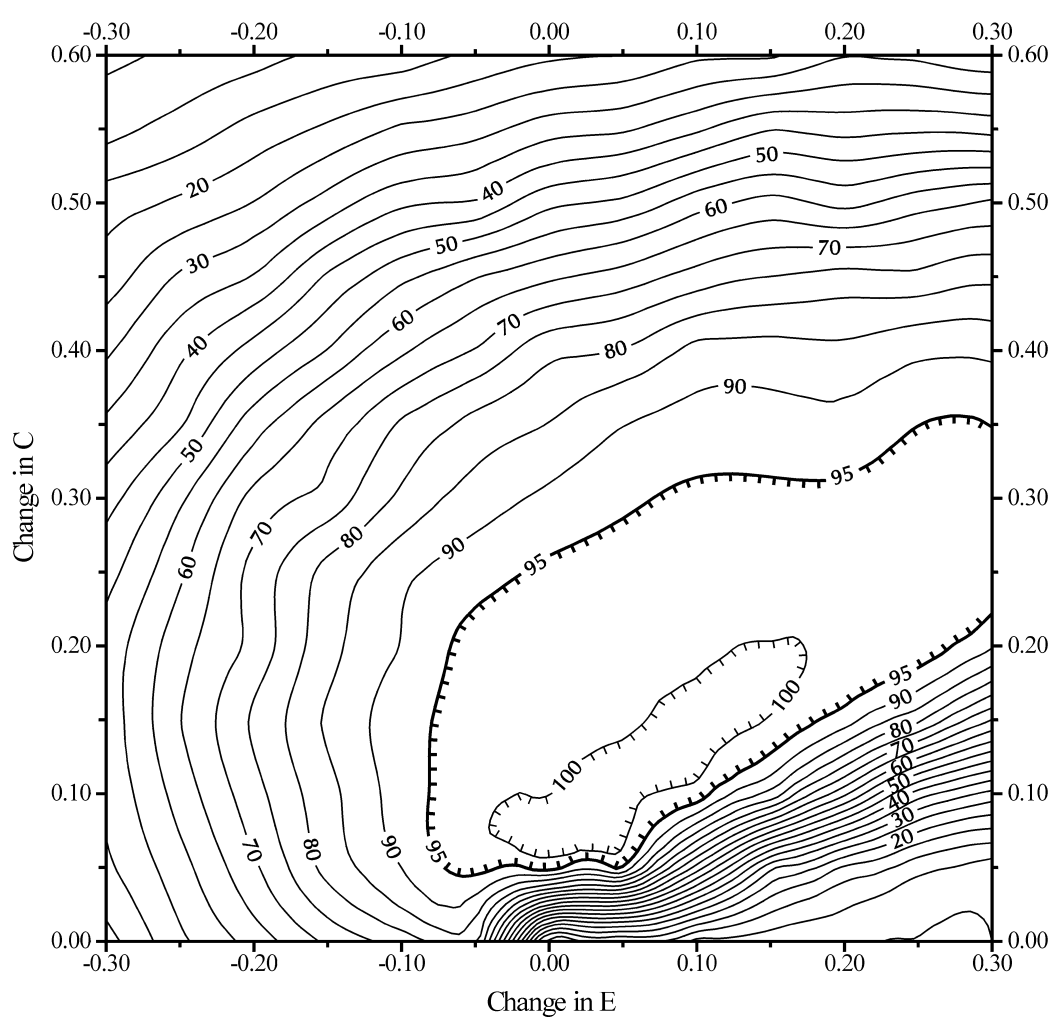

Fig. 3. Isopleth of percentage of positive PCYR outputs from 4000 simulation runs for various combinations of changes in $E$ and $C$. Hatched lines indicate the contour lines with percentage greater than 95\%, and darker line indicates the reference line to be used in Fig. 4.

in $E$ and $C$ that would have high probability to result in an increase of PCYR. The diagram shows that the percentage of positive PCYR was more sensitive to the change in $C$ than in $E$, especially in the beginning to change $C$ from the current level. Besides, we could see that the "area" to have $100 \%$ probability of producing positive PCYR was quite small. However, the "area" to have a minimum of $95 \%$ probability (hatched lines) was much larger, indicating that to obtain positive PCYR was comparatively easy.

Percentages of the "infeasible results" were also used as one of the references for selecting the best regulation option on $E$ and $C$. We took $5 \%$ as an ad hoc criterion or tolerant possibility of results being infeasible, for reference in this study. Information in Figs. 2 and 3 with the ad hoc tolerant criterion of infeasible results, can be summarized and combined into Fig. 4, in which the composite diagram of the selected con- tour lines are drawn. This gives an option combination picture with a tolerance of $5 \%$ being infeasible results. It implies that, to have at least $95 \%$ confidence that the output PCYR would be positive and its mean would be greater than $30 \%$, then the changes in $E$ should be regulated at somewhere between -0.05 and +0.15 and $C$ between +0.15 and +0.25 (the intercrossed shaded region).

We have also performed the same simulation as above but with a different assumption for input distribution. In this simulation, we adopted uniform distribution instead of triangular for comparison, and obtained the results in Fig. 5. No clear intercrossed region as in Fig. 4 could be found in Fig. 5 under the same conditions. We could only find a flat-shaped small region when the percentage of positive PCYR reduced to $90 \%$ or the percentage of mean PCYR reduced to $25 \%$. 


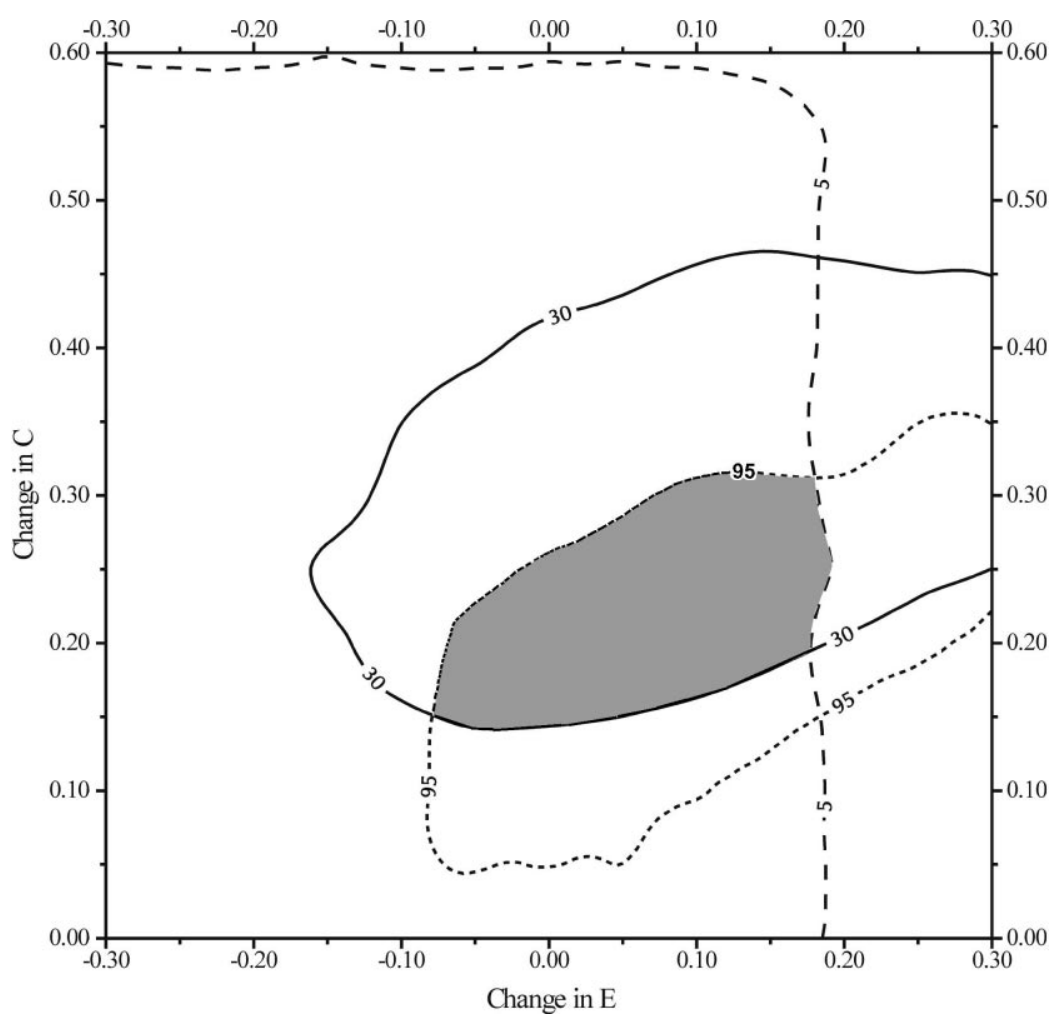

Fig. 4. Composite diagram of the selected contour lines from Figs. 2 and 3 against changes in $E$ and $C$. Solid contour line indicates the mean PCYR (30\%), dotted line the percentage of producing positive PCYR (95\%), and dashed line the percentage of producing infeasible results $(5 \%)$. See text for the implication of the shaded area.

\section{Discussion}

Uncertainty is inevitable in dynamic fishery systems. The mathematical estimates in the models for the systems and the data collected from the systems may involve such uncertainty and may cause bias in their estimation. Conditions for the data used, estimation approaches, and many other factors in different studies may also produce different estimations to the same stock. In general, there are two sources of bias common to most fisheries parameter estimation: the "errors in variables" where the independent variables (e.g., fishing effort, catches, size samples) of the estimation are not measured without error as is assumed; the "time-series bias" which arises from the time-series nature of fisheries data (Hilborn and Walters, 1992). The divergent estimates of parameters of the Indian Ocean albacore might result from both of these sources; they might also be caused by the socalled model misspecification.

In this study, only the uncertainty in the fishery parameters of Indian Ocean albacore estimated previously by different studies was considered, and no allowance has been made for the bias or variance of each parameter due to no sufficient available information. We simply present the idea that we have several estimates of a parameter but lack sufficient knowledge on the extent and the way these estimates were biased. The intention is to reach management advice with some confidence for the fish stock under this situation. Therefore we assumed that the "true" value of the parameter was occurred somewhere inside of the largest (upper range) and smallest (lower range) point estimates available, and then sample from the ranges (with some higher possibility of some values) as an input value to our model to draw the implication by 


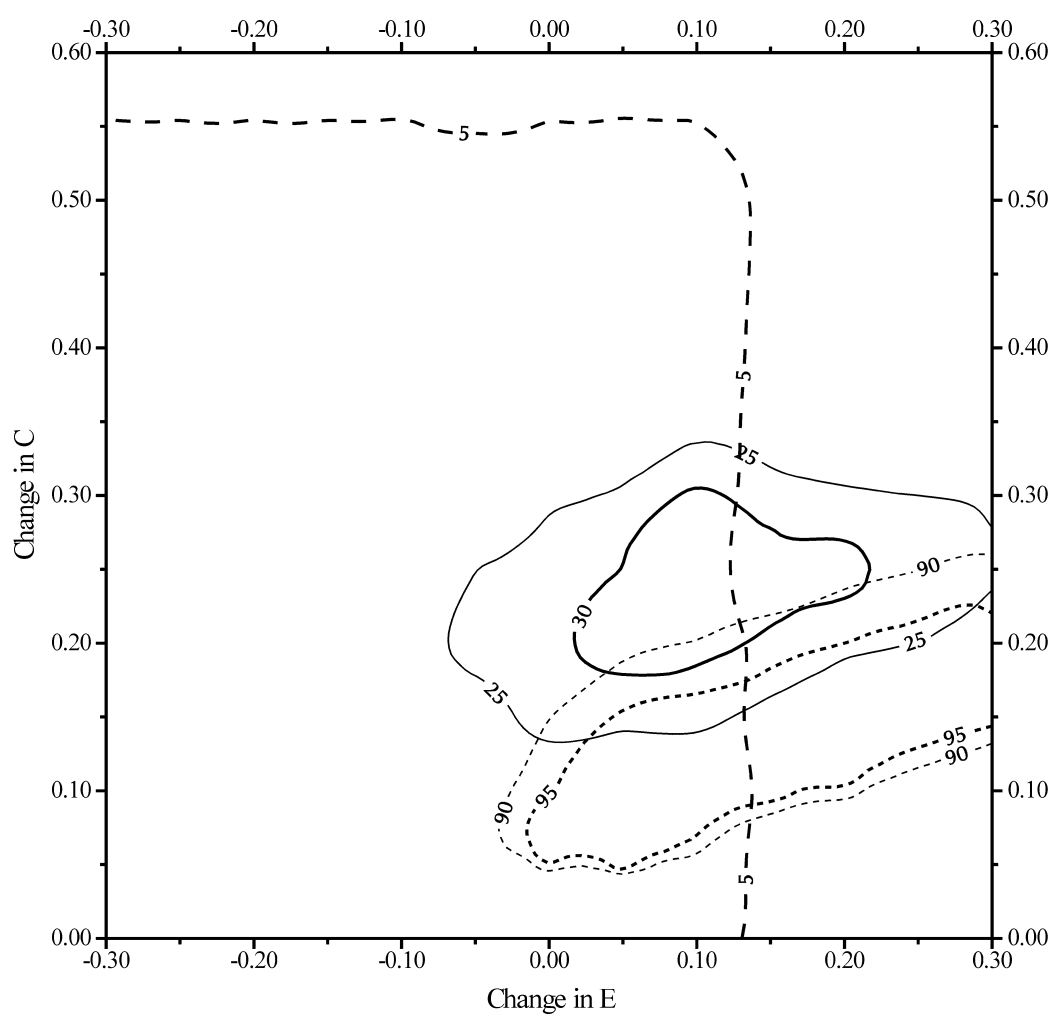

Fig. 5. Composite diagram of the selected contour lines from another simulation outputs of mean PCYR (light and dark solid lines), percentage of positive PCYR (light and dark dotted lines) and percentage of infeasible results (dashed line). The input parameters are assumed uniformly distributed, rather than triangularly as in Fig. 4.

changing the relative control parameters $(C$ or $E)$. We have assumed that those estimates were at least the hedge against the uncertainty. In this way the "true" uncertainty may be underestimated, however, it might be considered as an adapted approach and might be developed over time, as characteristics of the uncertainties are clarified or some of them are resolved.

From the results shown in Fig. 4, a simple management proposal on the stock to obtain large yield-perrecruit increase has derived. The proposal could be itemized as the following:

1. if the "true" values of the input parameters, i.e., $E, C$ and $M / K$, were located in somewhere between their ranges of previous estimates and were most likely located within the bound of the specified most possible values;

2. on the condition that the probability of obtaining feasible results was greater than $95 \%$;

3 . on the condition that the percentage of obtaining an increase in relative yield-per-recruit was greater than $95 \%$;

4. if the goal of a management was to increase the relative yield-per-recruit by an amount at least $30 \%$;

5. then, the suggestion for the regulation was to increase the relative selection size by an amount of $0.15-0.25$, and decrease the exploitation rate by an amount less than 0.05 or increase it by not more than 0.15 .

Based on this conclusion, manager may consider the most recent information or knowledge to select ad hoc estimates of $L_{\infty}$ and $M$, and then calculate to obtain the true control parameters of $l_{\mathrm{c}}$ and $F$ when necessary for management. In this sense, using $C$ and $E$ instead of $l_{\mathrm{c}}$ and $F$ as control parameter might leave more flexibility to managers in developing regulations in the face of uncertainty. A further consideration is needed when making management proposals based on 
the results, however, since the yield-per-recruit analysis does not make allowance of the effect of stockrecruitment. The analysis could provide a good longterm management option when there is no clear relationship between stock and recruits, but the option might not be appropriate if the stock has just suffered a recruitment failure (Pitcher and Hart, 1983).

For comparison, same simulation runs were also performed with a different assumption, i.e., uniform distribution, for the input uncertainty distribution. No clear conclusion could be drawn from the result on the same conditions as previous simulation. If the goal is reduced to obtain only $25 \%$ of yield-per-recruit increase, some regulatory options could still be found: the goal could be achieved by increasing the relative selection size by $0.13-0.15$, and exploitation rate by 0.05-0.13. However, due to the small conclusion area in Fig. 5 and the large coefficient of variance (CV) of the PCYR outputs (Table 2, discussed later), this option was rather unstable and needed to be more cautious in application.

We might also suppose a case that the regulation adopted was to change $C$ by 0.2 and $E$ by 0.1 , which was located in the shaded region of Fig. 4. In this case, the CV of the PCYR outputs, under the situation that the range of each input parameter been reduced to 75 , 50,25 and $1 \%$ of its original, could be calculated as in Table 2. This shows that the CVs based on uniform distribution were twice as much as those based on triangular distribution, which was coincided with the impression obtained above that the triangular distribution provided much stable results in this case. It is

\section{Table 2}

Coefficients of variation (CV) of the PCYR outputs of each simulated reduction in the uncertainty of the input parameters $(E, C$ and $M / K$ ), based on different input distributions (triangular and uniform distributions) ${ }^{\mathrm{a}}$

\begin{tabular}{llllllll}
\hline Fraction & \multicolumn{2}{l}{ Triangular distribution } & & \multicolumn{3}{c}{ Uniform distribution } \\
\cline { 2 - 3 } \cline { 7 - 8 } \cline { 6 - 8 } & $M / K$ & $C$ & $E$ & & $M / K$ & $C$ & $E$ \\
\hline 1.00 & 42.47 & 40.72 & 42.88 & & 87.72 & 88.35 & 90.41 \\
0.75 & 35.97 & 35.67 & 37.06 & & 85.03 & 73.41 & 78.89 \\
0.50 & 34.05 & 33.53 & 30.68 & & 85.51 & 56.71 & 69.07 \\
0.25 & 28.19 & 33.89 & 27.38 & & 83.59 & 51.43 & 65.16 \\
0.01 & 25.97 & 32.78 & 26.80 & & 83.48 & 50.89 & 67.80 \\
\hline
\end{tabular}

${ }^{\mathrm{a}}$ The simulated regulation is changing $C$ by 0.2 and $E$ by 0.1 . Numbers in the first column of the table is the fraction of the reduced parameter range to its original. worth noting that there is nothing wrong with either the triangular or uniform distributions, but when the ranges of the two are the same, they imply quite different levels of uncertainty; this makes the comparison on their results very difficult. Basically, it might be advisable: to consider results from uniform distribution in the case that parameter estimates vary widely and have no basis to help in selecting better values; to consider those from triangular distribution when some relevant knowledge are available for this species or from other similar species to be a reference for specifying more plausible values; to consider those from normal distribution when variances of the parameter estimates are available.

For the results of triangular distribution used in the present study, we also find from Table 2 that reducing uncertainty of the input parameters, except that of $C$, has resulted in great reduction in CV. The effect of reducing $C$ was great when its range was reduced to $75 \%$ of its original. Beyond that, however, the effect diminished. This was mainly because that its uncertainty range was not large $(0.20-0.46)$; a $25 \%$ reduction of its range has already greatly improved the computations.

On the other hand, improving the knowledge of $E$ has shown a significant effect on the reduction of $\mathrm{CV}$. Since the range of $M$ estimates was not so large (0.21-0.25), to reduce significantly the uncertainty of $E$ implied the necessity to largely reduce the uncertainty of $F$ in the end, which is quite troublesome in the dynamic fisheries.

Reducing uncertainty in $M / K$ has the most significant effect in reducing the variability of the output distribution, probably due to its large uncertainty (1.27-2.58). The CV has been greatly reduced when both the lower and upper bonds of its range narrowed or rescaled towards its mode. Based on these discussions, improving the knowledge of $E$ and $M / K$ was recommended for further studies to obtain results that are more precise.

In this study we did not take account of correlations between some of the parameter estimates. These correlations can be quite important sometimes, and when they are included, the uncertainty would be somewhat less. But correlations are not always easy to estimate. Take that between $L_{\infty}$ and $K$ for example; they should be correlated due to physiological reasons. But this correlation would be small comparing to their 
potentially high correlation when they are estimated simultaneously by nonlinear regression when there is not much contrast in the data. To do it correctly, we should examine the effects of both types of correlations separately, but it is not easy to discern between the two. In this regard, maybe we should call our treatment an "ad hoc method" with no well-understood ability to correct the bias from parameter correlations.

All the simulations in this study were based on the simple yield-per-recruit model, and the input parameters were all treated as independent variables, not as composites of others. This might cause losses of some important information from the individual parameter that constitutes the input parameter. Therefore, when more complete information is available to justify the use of extended seven-parameter model, it is worthwhile to conduct further similar study to treat the seven parameters separately and sample from separate distributions.

\section{Acknowledgements}

We would like to appreciate Dr. V.R. Restrepo, International Commission for the Conservation of Atlantic Tunas, Madrid, Spain, for his constructive suggestions on the simulation and discussion, and National Science Council for the financial support (No. NSC84-2321B-056-002). We also thank Dr. Y.H. Chien of National Taiwan Ocean University and the reviewers for their valuable comments.

\section{References}

Beverton, R.J.H., Holt, S.J., 1957. On the dynamics of exploited fish populations. Fish. Invest. Ser. II 19.
Beverton, R.J.H., Holt, S.J., 1966. Manual of methods for fish stock assessment. Part II. Tables of yield functions. FAO Fisheries Technical Paper 38.

Carlander, K.D., 1987. A history of scale age and growth studies of North American freshwater fish. In: Summerfelt, R.C., Hall, G.E. (Eds.), Age and Growth of Fish. Iowa State University Press, Ames, IA, pp. 3-14.

Casselman, J.M., 1983. Age and growth assessment of fish from their calcified structures - techniques and tools. In: Prince, E.D., Pulos, L.M. (Eds.), Proceedings of the International Workshop on Age Determination of Oceanic Pelagic Fishes: Tunas, Billfishes, and Sharks. NOAA Technical Report NMFS 8 , pp. $1-17$.

Chang, S.-K., 1993. Stock assessment of Indian albacore using nonequilibrium production model, Ph.D. Dissertation. Institute of Oceanography, National Taiwan University, 90 pp.

Chang, S.-K., Hsu, C.-C., 1992. Nonlinear estimation on von Bertalanffy growth parameters of Atlantic albacore, Thunnus alalunga. Acta Oceanogr. Taiwanica 29, 59-71.

Chang, S.-K., Liu, H.-C., Hsu, C.-C., 1993. Estimation of vital parameters for Indian albacore through length-frequency data. J. Fish. Soc. Taiwan 20 (1), 1-17.

Hilborn, R., 1979. Comparison of fisheries control systems that utilize catch and effort data. J. Fish. Res. Bd. Can. 36, 14771489.

Hilborn, R., Walters, C.J., 1992. Quantitative Fisheries Stock Assessment. Chapman \& Hall, New York, 570 pp.

Lee, Y.-C., Liu, H.-C., 1992. Age determination, by vertebra reading, in Indian albacore, Thunnus alalunga (Bonnaterre). J. Fish. Soc. Taiwan 19 (2), 89-102.

Liu, H.-C., Lee, Y.-C., 1992. The yield per recruit analysis for Indian Ocean albacore stock. The Third Asian Fisheries Forum, Singapore, October 26-30, 1992, Asian Fisheries Society, 4 pp.

Ludwig, D., 1981. Harvesting strategies for a randomly fluctuating population. J. Const. Int. Explor. Mer. 39, 168-174.

Majkowski, J., Hampton, J., 1983. The effect of parameter uncertainties in an age-length relationship upon estimating the age composition of catches. Can. J. Fish. Aquat. Sci. 40, 272-280.

Pitcher, T.J., Hart, P.J.B., 1983. Fisheries Ecology (reprint with corrections). The AVI Publication Co.

Restrepo, V.R., Fox, W.W., 1988. Parameter uncertainty and simple yield-per-recruit analysis. Trans. Amer. Fish. Soc. 117, 282 289. 